\title{
Clustering of ions at atomic dimensions in quantum plasmas
}

\author{
PADMA K. SHUKLA ${ }^{1,2}$ and BENGT ELIAS S ON ${ }^{1}$ \\ ${ }^{1}$ International Centre for Advanced Studies in Physical Sciences \& Institute for Theoretical Physics, \\ Faculty of Physics \& Astronomy, Ruhr-University Bochum, D-44780 Bochum, Germany \\ (profshukla@yahoo.de) \\ ${ }^{2}$ Department of Mechanical and Aerospace Engineering \& Center for Energy Research, \\ University of California San Diego, La Jolla, CA 92093, USA
}

(Received 28 October 2012; revised 19 November 2012; accepted 20 November 2012; first published online 20 December 2012)

\begin{abstract}
By means of particle simulations of the equations of motion for ions interacting among themselves under the influence of newly discovered ShuklaEliasson attractive force (SEAF) in a dense quantum plasma, we demonstrate that the SEAF can bring ions closer at atomic dimensions. We present simulation results of the dynamics of an ensemble of ions in the presence of the SEAF without and with confining external potentials and collisions between ions and degenerate electrons. Our particle simulations reveal that under the SEAF, ions attract each other, come closer, and form ionic clusters in the bath of degenerate electrons that shield ions. Furthermore, an external confining potential produces robust ion clusters that can have cigar- and ball-like shapes, which remain stable when the confining potential is removed. The stability of ion clusters is discussed. Our results may have applications to solid density plasmas (density exceeding $10^{23}$ per $\mathrm{cm}^{3}$ ), where the electrons will be degenerate and quantum forces due to the electron recoil effect caused by the overlapping of electron wave functions and electron tunneling through the Bohm potential, electron-exchange and electron-exchange and electron correlations associated with electron- $1 / 2$ spin effect, and the quantum statistical pressure of the degenerate electrons play a decisive role.
\end{abstract}

\section{Introduction}

During the early 1930s, there were several discoveries related to non-Coulombic shielded potential distributions that exhibit the role of collective interactions between electrons and ions in electro-chemistry (Debye and Hückel 1923) (viz. electrolytes and colloidal suspensions), solid state (Fermi 1927; Thomas 1927), and gaseous (Langmuir 1929) plasmas, and between neutrons and protons in elementary particle physics (Yukawa 1935). The screened non-Coulombic potentials, which were obtained by using linearized theory based on the assumption that the potential energy between the particles is much smaller than the particle kinetic energy, are now known as the Debye-Hückel (DH), Thomas-Fermi (TF), and Yukawa potentials in the context of electrochemistry and plasma physics, condensed matter physics, and nuclear physics respectively. The DH, TF, and Yukawa potentials describe short-range (of the order of the $\mathrm{DH}$ radius, the TF radius, and the Yukawa radius, which are fixed by the size of a shielded cloud) repulsive interactions between two particles that have the same polarity. The DH theory has also been extended to dusty plasma physics where charged dust particles are shielded by non-degenerate electrons and ions. The $\mathrm{DH}, \mathrm{TF}$, and Yukawa interaction potentials, which significantly deviate from the long-range Coulomb interaction potential, have important applications to the understanding of phase transitions (Kremer et al. 1986; Avinash 2007; Klumov 2010) in the different areas of physical sciences.

In order for charged particles to form ordered structures under the influence of Coulombic, DH, TF, and Yukawa repulsive forces, one must confine the likecharged particles in an external potential so as to bring them to a minimum energy state. Examples include the Wigner crystals (Wigner 1934) composed of an ensemble of electrons on the surface of liquid helium, ion crystals in laser cooled Paul (Drewsen et al. 1998) and Penning (electromagnetic) traps (Tan et al. 1995), charged dust particle crystals (Wuerker et al. 1959), which were formed when like-charged dust particles were kept together via external confining potentials despite short-range Coulombic or shielded Coulombic repulsive forces between charged dust particles. In fact, both electron and ion crystals, as well as crystals of colloidal suspensions and oil droplets have been observed experimentally under different physical circumstances (Berg and Gaukler 1969; Crandall and Williams 1971; Grimes and Adams 1979; Winter and Ortjohann 1991; Tan et al. 1995; Drewsen et al. 1998; Robertson and Younger 1999; Mølhave and Drewsen 2000; Deshpande and Bockrath 2008; Staanum et al. 2010). Moreover, an ensemble of strongly correlated micron-sized negative dust particles form dust Coulomb crystals (Chu and I 
1994; Hayashi and Tachibana 1994; Thomas et al. 1994; Barkan and Merlino 1995; Fortov et al. 1997; Mohideen et al. 1998) when they were confined by the sheath parabolic potential in low-temperature laboratory dusty plasma discharges (Shukla and Mamun 2002; Shukla and Eliasson 2009). The condensation of charged dust particles occurs since the dusty plasma $\Gamma_{d}$ (the ratio between the Coulomb energy between highly charged dust grains and the average dust particle kinetic energy) becomes relatively large due to the high dust charge state and low dust temperature. The attraction between like-charged dust particles forming dust Coulomb crystals may also be attributed to attractive forces arising from overlapping of the dusty plasma Debye spheres (Resendes et al. 1998), ion focusing and wakefield effects (Nambu et al. 1995; Vladimirov and Nambu 1995; Shukla and Rao 1996), and dust dipoledipole interactions (Mohideen et al. 1998; Shukla and Mamun 2002; Shukla and Eliasson 2009). The Cooper's pairing of charged dust particles, which are glued by ions, led to the discovery of a soft-condensed matter of dust particle crystals in low-density and low-temperature classical plasma with Maxwell-Boltzmann distributions for electrons and ions. It turns out that several milestones were reached in the areas of ordered crystalline structures composed of charged particles (e.g. an ensemble of electrons, ions, as well as charged colloidal and dust particles in low-temperature physical systems) in physical systems, which share some common physics.

However, solid density plasmas are of fundamental importance for industrial applications (e.g. semiconductors, nano-diodes, and metallic nanostructures for thin films), for inertial confinement fusion (ICF) schemes that utilizes high-density compressed (HDC) plasmas, as well as for planetary systems (e.g. the core of Jupiter (Fortov 2009)) and superdense astrophysical objects (e.g the cores of white dwarf stars, warm dense matter). In dense plasmas, one has to account for degeneracy (Chandrasekhar 1931, 1939) of electrons that obey the Fermi-Dirac distribution function. Correspondingly, quantum mechanical effects play a vital role, since in such dense plasmas the Wigner-Seitz radius $d=$ $\left(3 / 4 \pi n_{0}\right)^{1 / 3}$ is comparable to the thermal de Broglie wavelength, $\lambda_{B}=\hbar / m V_{T}$ (which is a measure of the extent of electron wave functions), where $\hbar$ is Planck's constant divided by $2 \pi, m$ is the electron mass, $V_{T}=$ $\sqrt{k_{B} T / m}$, the electron thermal speed due to random electron motions, and $k_{B}$ is the Boltzmann constant. Also, in dense plasmas with degenerate electrons, $\lambda_{B}$ turns out to be much smaller than the Landau length, $\lambda_{L}=e^{2} / k_{B} T$, which can be conveniently expressed as $k_{B} T \ll e^{2} / a_{B}$, where $a_{B}=\hbar^{2} / m e^{2}$ is the Bohr radius of a hydrogen atom. The electron degeneracy effects at nanoscales in dense plasmas can thus be captured through the consideration of the Fermi-Dirac statistics for electrons with spin-1/2 (Fermions), and overlapping of electron wavefunctions due to Heisenberg's uncertainty principle and Pauli's exclusion principle, as well as electron-exchange and electron-correlations. Hence, there are quantum forces (Chandrasekhar 1931, 1939; Wilhelm 1971; Gardner and Ringhofer 1996; Manfredi and Haas 2001; Manfredi 2005; Shukla and Eliasson 2006, 2007; Shaikh and Shukla 2007; Brodin et al. 2008; Crouseilles et al. 2008; Melrose 2008; Tsintsadze and Tsintsadze 2009; Shukla and Eliasson 2010, 2011; Haas 2011; Mendonça 2011; Vladimirov and Tyshetskiy 2011) associated with the quantum statistical electron pressure (Chandrasekhar 1931, 1939; Landau and Lifshitz 1980), the quantum Bohm potential (Wilhelm 1971; Gardner and Ringhofer 1996; Manfredi and Haas 2001; Manfredi 2005; Shukla and Eliasson 2010, 2011; Haas 2011) through which degenerate electrons can tunnel through (often known as the quantum electron recoil effect), as well as the electron-exchange and electron-correlation potentials (Hedin and Lundqvist 1971; Brey et al. 1990; Crouseilles et al. 2008). It has been found that the above-mentioned quantum forces acting on degenerate electrons in quantum plasmas introduce new dispersive features to electron plasma oscillations (EPOs) (Bohm 1952; Klimontovich and Silin 1952a,b; Bohm and Pines 1953 ) with frequencies in the x-ray regime, which can be assessed by using collective $\mathrm{x}$-ray spectroscopic scattering techniques (Glenzer et al. 2007; Glenzer and Redmer 2009). In fact, Glenzer et al. (2007) have reported EPOs in warm dense matter (with the peak electron number density $\sim 3 \times 10^{23} \mathrm{~cm}^{-3}$ and the equilibrium electron and ion temperatures of $12 \mathrm{eV}\left(\sim 1.5 \times 10^{5}\right.$ degrees Kelvin). The latter is different from the electron Fermi temperature, $T_{F}=\left(\hbar^{2} / 2 k_{B} m_{e}\right)\left(3 \pi^{2} n_{0}\right)^{2 / 3} \approx 1.7 \times 10^{5}$ degrees Kelvin corresponding to an electron number density $n_{0} \approx 2.5 \times 10^{23} \mathrm{~cm}^{-3}$. Thus, the recent experiments of Glenzer et al. (2007) have unambiguously demonstrated the importance of the quantum statistical pressure and quantum electron recoil effects on the frequency spectra of EPOs (Bohm 1952; Klimontovich and Silin 1952a,b; Bohm and Pines 1953; Shukla and Eliasson 2010, 2011), although a previous experimental investigation (Watanabe 1956) had already established the quantum dispersion properties of EPOs in metals.

Very recently, Shukla and Eliasson $(2012 a, b, c)$ discovered an oscillating shielded Coulomb (OSC) potential (also referred to as the Shukla-Eliasson attractive potential (SEAP) (Akbari-Moghanjoughi 2012)), which is valid for the potential energy much smaller than $k_{B} T$ and $m c^{2}$, around a stationary test ion in an unmagnetized quantum plasma, where $c$ is the speed of light in vacuum. The SEAP arises due to collective interactions between an ensemble of degenerate electrons that shield an isolated ion at atomic dimensions. The profile of the SEAP in quantum plasmas resembles the Lennard-Jones (LJ) potential in atomic gases. The Shukla-Eliasson attractive force (SEAF), defined as minus the gradient of the SEAP, brings ions closer to form ion clusters in quantum plasmas. In this paper, we demonstrate the formation of ion clusters at atomic dimensions by performing computer simulations of the equations of 


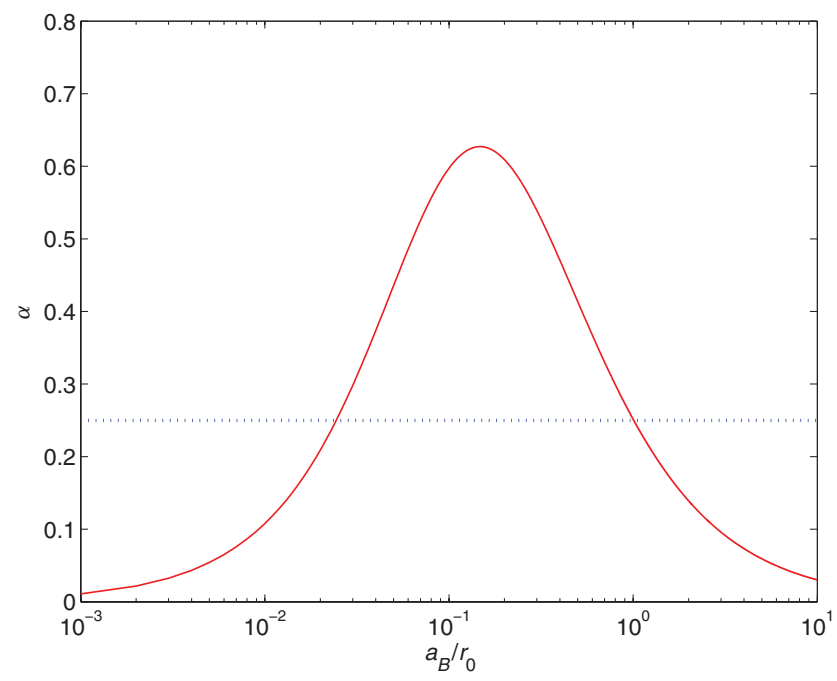

Figure 1. (Colour online) The value of $\alpha$ as a function of $a_{B} / r_{0}$. The critical value $\alpha=1 / 4$ is indicated with a dotted line.

motion for an ensemble of ions that are interacting with each other through the SEAF.

\section{The SEAF}

The existence of the SEAP, which is obtained from Fourier transformation of Poisson's equation with the quasistationary electron density perturbation deduced from the linearized continuity and generalized momentum equation (Shukla and Eliasson 2012a,b,c) for nonrelativistic, degenerate electrons in a dense quantum plasma, critically depends on the electron number density through the parameter $\alpha=\hbar^{2} \omega_{p e}^{2} / 4 m^{2} u_{*}^{4}$, where $u_{*}=\left(v_{*}^{2} / 3+v_{e x}^{2}\right)^{1 / 2}$. The parameter $\alpha$ measures the quantum electron recoil effect caused by the quantum Bohm potential (Wilhelm 1971; Gardner and Ringhofer 1996; Manfredi and Haas 2001; Manfredi 2005) $V_{B}=$ $\left(\hbar^{2} / 2 m\right)(1 / \sqrt{n}) \nabla^{2} \sqrt{n}$ compared to the quantum statistical Fermi electron pressure and the electron-exchange and electron-correlation effects arising from 1/2-spin of degenerate electrons. Here $v_{*}=\hbar\left(3 \pi^{2}\right)^{1 / 3} / m r_{0}$ is the electron Fermi speed and $v_{e x}=\left(0.328 e^{2} / m r_{0}\right)^{1 / 2}[1+$ $\left.0.62 /\left(1+18.36 a_{B} n_{0}^{1 / 3}\right)\right]^{1 / 2}$ includes the effects of electron exchange and electron correlations, where $r_{0}=n_{0}^{-1 / 3}$ represents the average inter-electron distance. The expression for $v_{e x}$ is derived by linearizing the sum of the electron exchange and electron correlation potentials (Hedin and Lundqvist 1971; Brey et al. 1990) $V_{x c}=$ $0.985 e^{2} n^{1 / 3}\left[1+\left(0.034 / a_{B} n^{1 / 3}\right) \ln \left(1+18.37 a_{B} n^{1 / 3}\right)\right]$. We note that $\alpha$ depends only on $a_{B} / r_{0}$, as $\alpha \simeq 9.3 \pi\left(a_{B} / r_{0}\right) /$ $\left[1+\left(3 \pi^{2}\right)^{2 / 3}\left(a_{B} / r_{0}\right)+0.62 /\left(1+18.36 a_{B} / r_{0}\right)\right]^{2}$. Shukla and Eliasson $(2012 \mathrm{a}, \mathrm{b}, \mathrm{c})$ found that attractive potentials between ions exist only for $\alpha>1 / 4$. Figure 1 displays the value of $\alpha$ as a function of $a_{B} / r_{0}$, where one observes that it is above a critical value 0.25 only for a limited range $2 \times 10^{-2}<a_{B} / r_{0}<1$, corresponding to an electron number density in the range $5.4 \times 10^{19} \mathrm{~cm}^{-3}<n_{0}<$

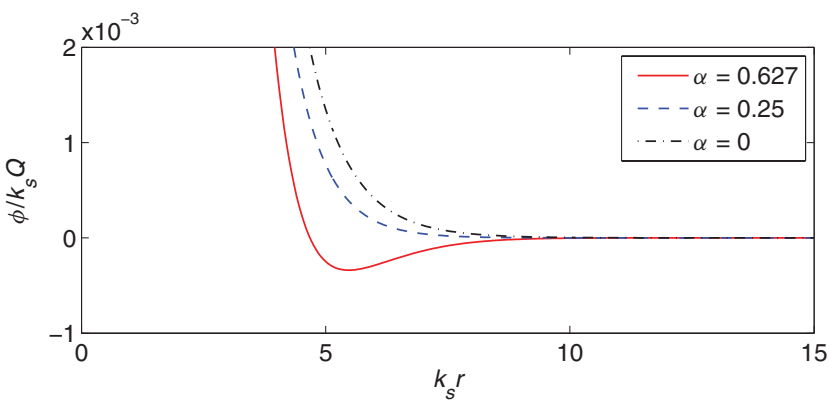

Figure 2. (Color online) The electric potential $\phi$ as a function of $r$ for $\alpha=0.627$ (solid curve), $\alpha=0.25$ (dashed curve), and $\alpha=0$ (dash-dotted curve). The value 0.627 is the maximum possible value of $\alpha$ in our model, obtained for $a_{B} / r_{0} \approx 0.15$.

$6.7 \times 10^{24} \mathrm{~cm}^{-3}$ (with $a_{B}=5.3 \times 10^{-9} \mathrm{~cm}$ ). The maximum value is $\alpha \approx 0.627$ at $a_{B} / r_{0} \approx 0.15$, corresponding to the electron number density $n_{0} \approx 2 \times 10^{22} \mathrm{~cm}^{-3}$, a few times below solid densities. The validity of the SEAP has been further expanded (Akbari-Moghanjoughi 2012) for wider density ranges by including Chandrasekhar's generalized pressure law (Chandrasekhar 1931, 1939) for degenerate electron fluids and Salpeter's (1961) electronexchange and electron-correlation potentials that are of astrophysical interest (e.g. the cores of white dwarf stars). The nonlinear shielding effects on the SEAF is discussed by Shukla et al. (2012).

For $\alpha>0.25$, the profile of electric potential as a function of distance $r$ around a stationary test ion charge $Q$ is (Shukla and Eliasson 2012a,b,c)

$$
\phi(\mathbf{r})=\frac{Q}{r}\left[\cos \left(k_{i} r\right)+b_{*} \sin \left(k_{i} r\right)\right] \exp \left(-k_{r} r\right),
$$

which is referred to as the SEAP. Here $Q$ is the ion charge, $b_{*}=1 / \sqrt{4 \alpha-1}, k_{i}=\left(k_{s} / \sqrt{4 \alpha}\right)(\sqrt{4 \alpha}-1)^{1 / 2}$, and $k_{r}=\left(k_{s} / \sqrt{4 \alpha}\right)(\sqrt{4 \alpha}+1)^{1 / 2}$, with $k_{s}=\omega_{p e} / u_{*}$ being the modified inverse TF screening length. The spatial profile of the SEAP in Fig. 2 shows a distinct minimum for the case $\alpha=0.627$. The negative part of the SEAP, given by (1), resembles the LJ potential and leads to a shortrange SEAF between neighboring ions. On the other hand, the potential distribution around a test ion for $\alpha<0.25$ reads (Shukla and Eliasson 2012a,b,c)

$$
\phi(\mathbf{r})=\frac{Q}{2 r}\left[(1+b) \exp \left(-k_{+} r\right)+(1-b) \exp \left(-k_{-} r\right)\right],
$$

where $b=1 / \sqrt{1-4 \alpha}$ and $k_{ \pm}=k_{s}(1 \mp \sqrt{1-4 \alpha})^{1 / 2} / \sqrt{2 \alpha}$. For this case, the potential is positive and monotonically decreasing (cf. Fig. 2), giving rise to a repulsive force only (similar to the TF force) between ions. In the high- or low-density limit, where $\alpha \rightarrow 0$, we recover the modified TF screened Coulomb potential $\phi(\mathbf{r})=(Q / r) \exp \left(-k_{s} r\right)$.

\section{Demonstration of ion clustering}

We here present a computer simulation study of the dynamics of a system of ions interacting with each other under the action of the SEAF. For this purpose, we numerically solve the equations of motion for a system 


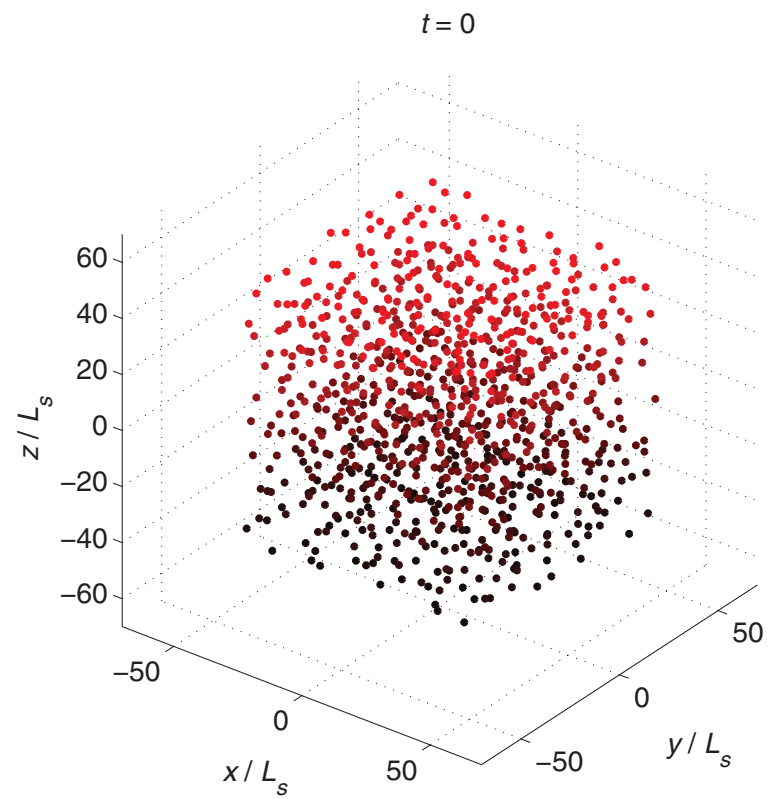

Figure 3. (Color online) The initial positions of ions in particle simulations.

of ions with equal charges and masses, given by

$$
M \frac{d \mathbf{v}_{j}}{d t}=-Q \sum_{i \neq j} \nabla_{j} \phi\left(\left|\mathbf{R}_{i j}\right|\right)-\nabla_{j} V_{c}\left(\mathbf{r}_{j}\right)-M v \mathbf{v}_{j}
$$

where instantaneous position of each ion is determined from $d \mathbf{r}_{j} / d t=\mathbf{v}_{j}$. Here $\mathbf{R}_{i j}=\mathbf{r}_{i}-\mathbf{r}_{j}$ is the radius vector between particle $i$ and $j, \mathbf{r}_{j}(t)$ is the position and $\mathbf{v}_{j}(t)$ is the velocity of the $j$ th ion, $M$ is the ion mass, $\nabla_{j}$ denotes the gradient of $\phi$ at position $\mathbf{r}_{j}$, and $v$ denotes an effective collision frequency, which tends to retard the ion motion. The external confining potential, $V_{c}(\mathbf{r})=(M / 2)\left(\omega_{\perp}^{2} r_{\perp}^{2}+\omega_{z}^{2} z^{2}\right)$, of charged particles may

(a)

$$
t=15000 \tau
$$

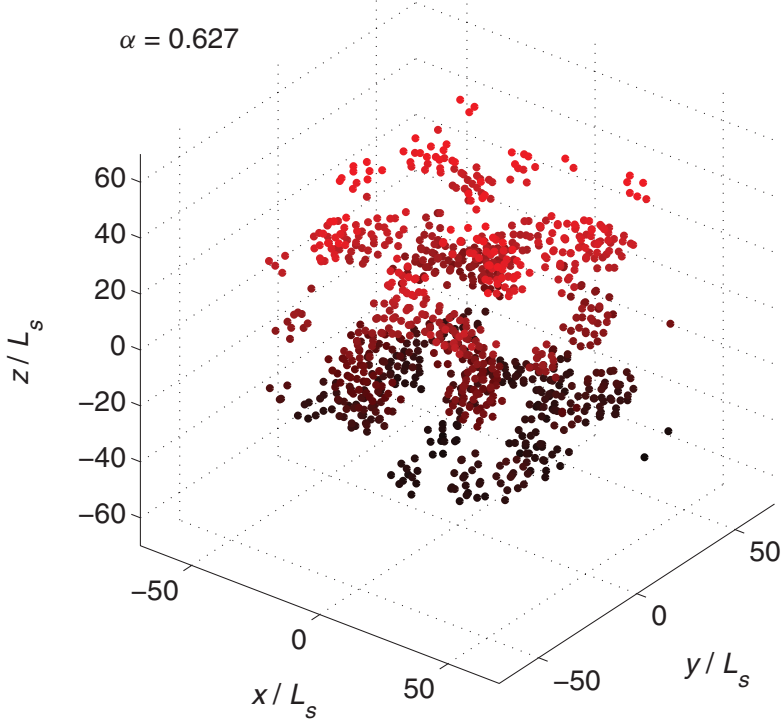

have different amplitudes $\omega_{\perp}$ and $\omega_{z}$ perpendicular and parallel to the $z$-axis, respectively, where $r_{\perp}^{2}=r_{x}^{2}+r_{y}^{2}$.

In order to demonstrate the clustering of ions under the SEAF, we now carry out particle simulations of (3) with 1000 particles, initially randomly placed in space, as shown in Fig. 3. In the first set of simulations, displayed in Fig. 4, we consider interactions of the ions in the absence of the external confining potential $V_{c}$, viz. $\omega_{z}=\omega_{\perp}=0$, and with $v=0.01 \tau^{-1}$. The positions of ions at the end of the simulations are shown in Fig. 4 at time $t=15000 \tau$. Here the positions and time are in units of $L_{s}=k_{s}^{-1}$ and $\tau=M^{1 / 2} / Q k_{s}^{3 / 2}=$ $(\pi / 16)^{1 / 8}\left(a_{B} / r_{0}\right)^{3 / 8} \omega_{p i}^{-1} / \alpha^{3 / 8} Z_{i}$, respectively, where $\omega_{p i}=$ $\left(4 \pi Q^{2} n_{i 0} / M\right)^{1 / 2}$ is the ion plasma frequency, and $n_{i 0}$ is the equilibrium ion number density, related to the electron number density $n_{0}$ via the quasi-neutrality condition $Z_{i} n_{i 0}=n_{0}$, where $Z_{i}$ is the ion charge state. For $\alpha=$ 0.627 , which leads to a potential minimum (cf. Fig. 2), we observe the clustering of ions and the formation of large-scale ionic structures. The clustering of ions is a relatively slow process in comparison with the ion plasma period $2 \pi / \omega_{p i}$. Ion pairs and smaller clusters are initially formed, and later the larger ion clusters are gradually formed by the agglomeration of smaller ion clusters. As a contrast, for $\alpha=0.25$, we see in Fig. 4 that there does not exist condensation/coalescence of ions. This is due to the fact that there is no potential minimum for this value of $\alpha$ (cf. Fig. 2), and hence the inter-ion force is always repulsive for this case.

Furthermore, we have carried out a set of simulations with a symmetric potential $V_{c}$ with $\omega_{\perp}=\omega_{z}=2 \times$ $10^{-3} \tau^{-1}$. Here, as seen in Fig. 5, almost spherical nonCoulombic ion crystals are formed for both $\alpha=0.627$ and 0.25 . We also performed simulations, including an asymmetric external potential $V_{c}$ with $\omega_{\perp}=6 \times 10^{-3} \tau^{-1}$

$$
t=15000 \tau
$$

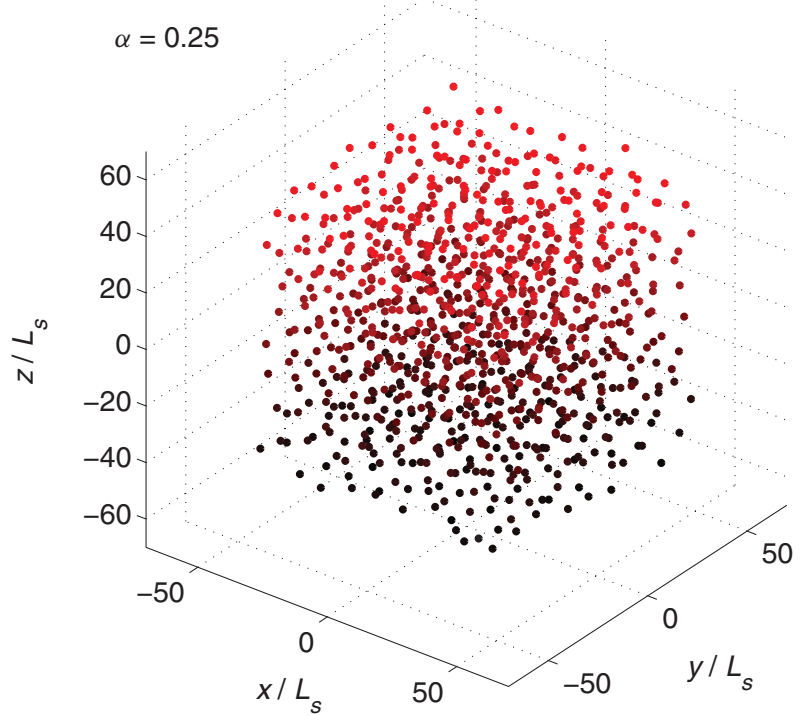

Figure 4. (Color online) The positions of ions $t=15000$ for (a) $\alpha=0.627$ and (b) $\alpha=0.25$, showing the clustering and solidification of ions for $\alpha=0.627$, but not for $\alpha=0.25$. 
(a)

$$
t=15000 \tau
$$

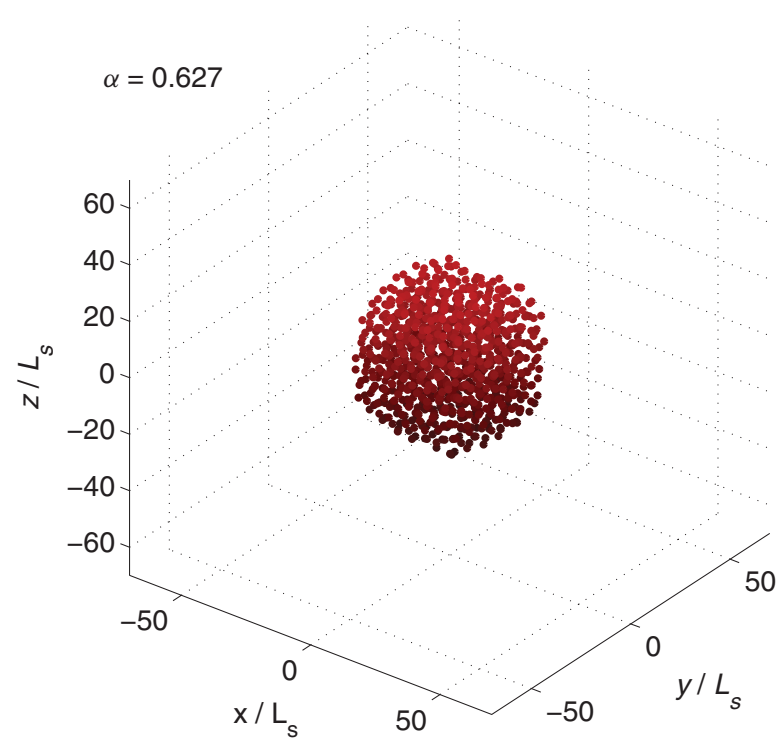

(b) $t=15000 \tau$

Figure 5. (Color online) The positions of ions at $t=15000$ for (a) $\alpha=0.627$ and (b) $\alpha=0.25$, including a symmetric parabolic potential with $\omega_{\perp}=\omega_{z}=2 \times 10^{-3} \tau^{-1}$. Almost spherical non-Coulombic ion crystals are formed.

(a)

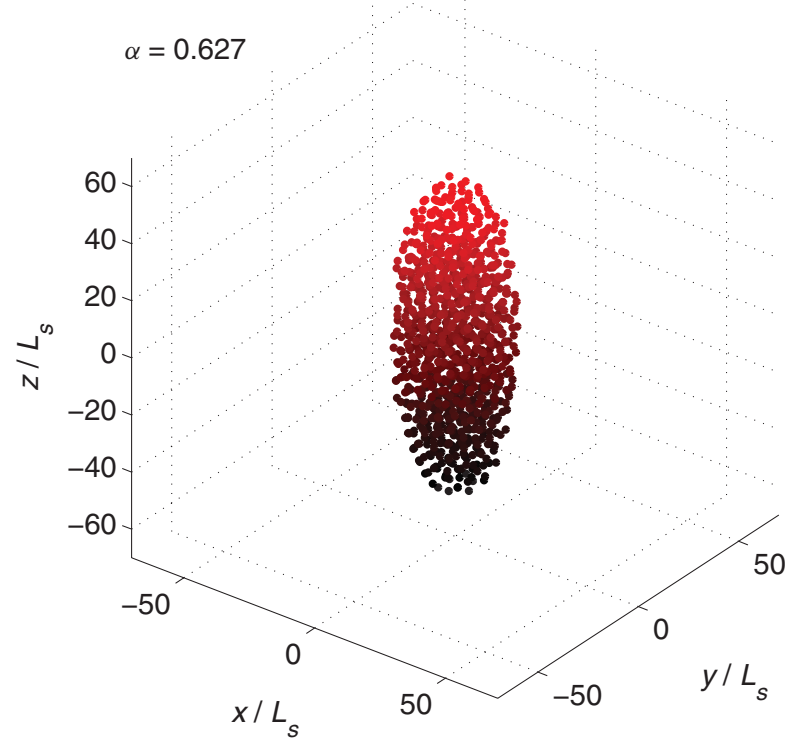

(b) $t=15000 \tau$

Figure 6. (Color online) The positions of ions at $t=15000$ for (a) $\alpha=0.627$ and (b) $\alpha=0.25$, including an asymmetric parabolic potential with $\omega_{\perp}=6 \times 10^{-3} \tau^{-1}$ and $\omega_{z}=2 \times 10^{-3} \tau^{-1}$. Here elongated non-Coulombic ion crystals are formed.

and $\omega_{z}=2 \times 10^{-3} \tau^{-1}$, providing a stronger confinement in the perpendicular direction. Figure 6 displays the final state for $\alpha=0.627$ and 0.25 , where ions, in both cases, form non-Coulombic ion crystals elongated along the $z$-direction. In general, the formation of non-Coulombic ion crystals is attributed to the balance between the external and inter-ion potentials, where the system tends to a configuration of a minimum potential energy. We note that similar configurations have been previously reported for both charged macro-particles (Wuerker et al. 1959) and ions (Drewsen et al. 1998; Mølhave and Drewsen 2000; Staanum et al. 2010) confined by external potentials in the Paul trap. Finally, in Figs. 7 and 8, we continued the simulations in Figs. 5 and 6 and set the external confining potential to zero at time $t=15000 \tau$. For both cases, with symmetric and asymmetric potentials, we see that ion crystals remained tightly packed for $\alpha=0.627$, where it performed damped oscillations as elastic solids before settling down to the final states in Figs. 7(a) and 8(a). In contrast, for $\alpha=0.25$, the ion cloud expanded to larger and less dense ion clouds as seen in Figs. 7(b) and 8(b) at 
(a) $\quad t=20000 \tau$

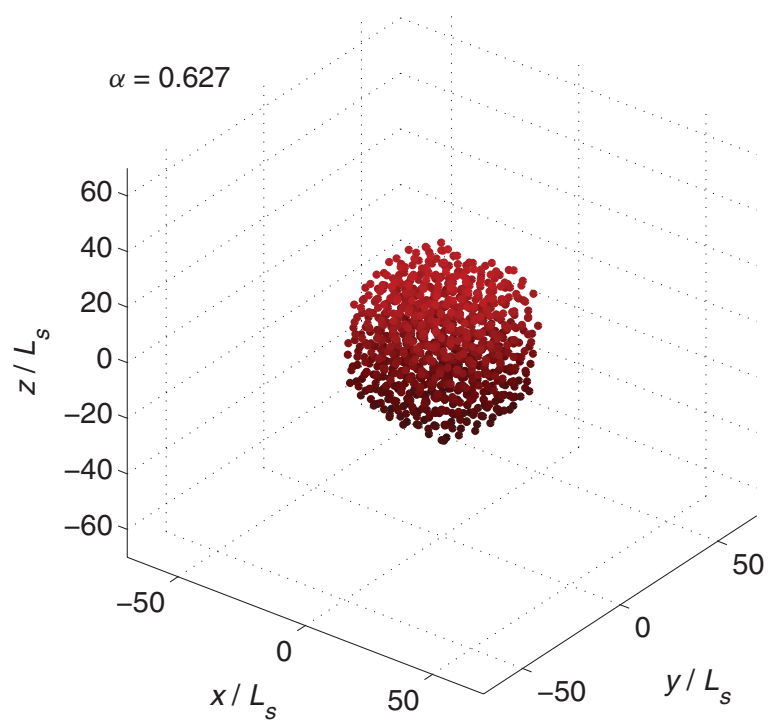

(b)

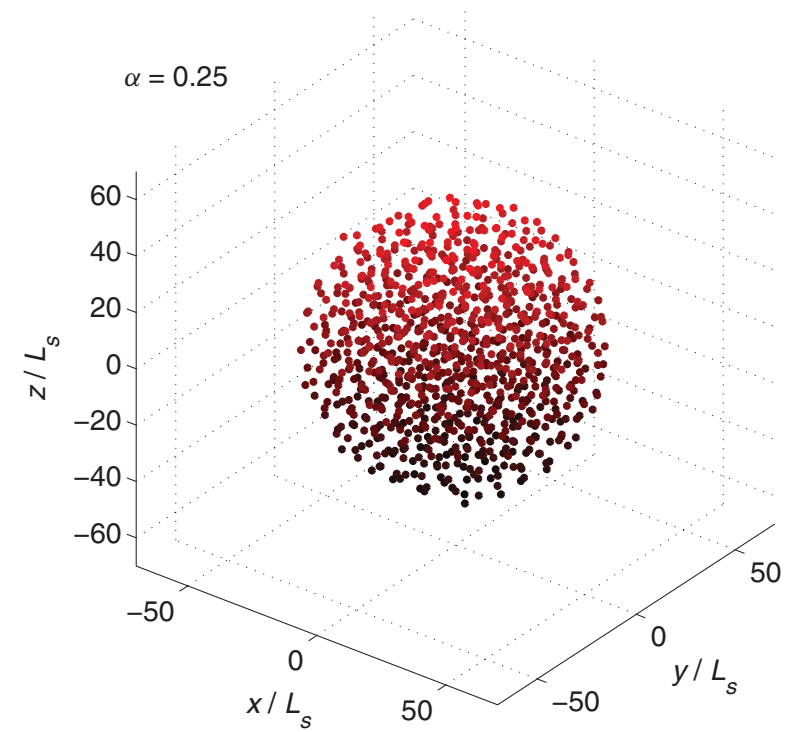

Figure 7. (Color online) The positions of ions at $t=20000 \tau$ for (a) $\alpha=0.627$ and (b) $\alpha=0.25$ for the case of a symmetric parabolic potential in Fig. 5, where the potential is set to zero at $t=15000 \tau$. The ion cluster remains tightly packed for $\alpha=0.627$, while the ion cloud expands for $\alpha=0.25$.

(a)

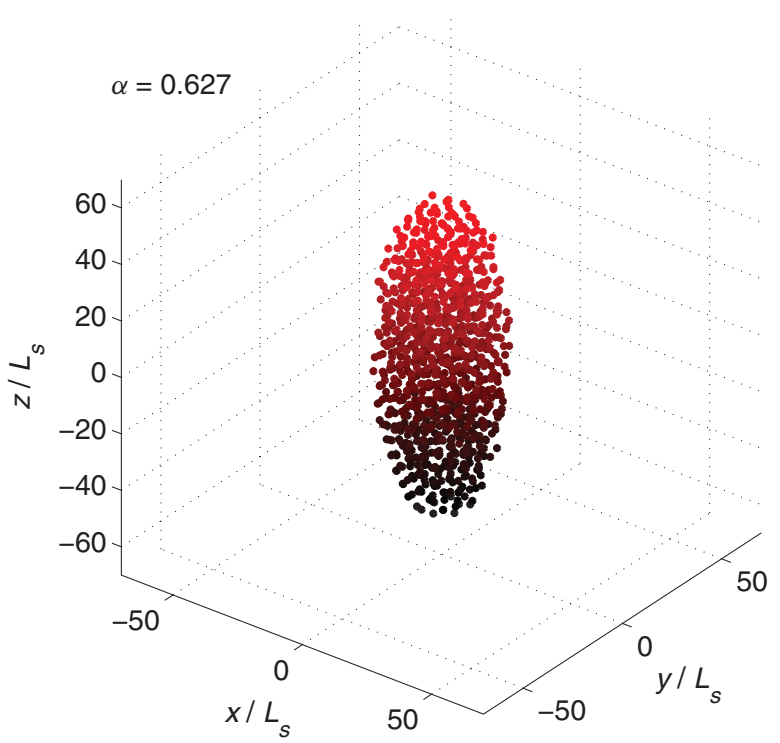

(b)

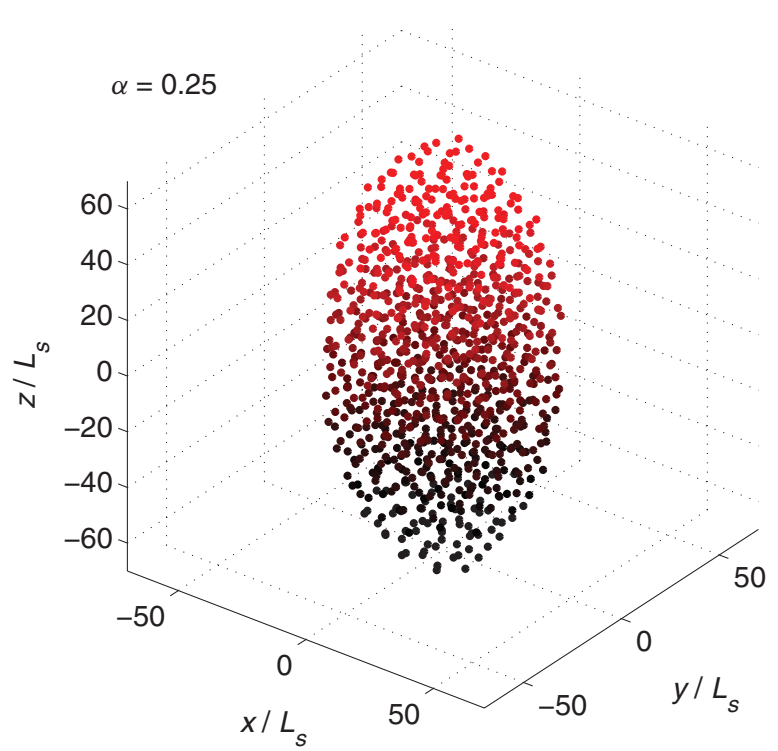

Figure 8. (Color online) The positions of ions at $t=20000 \tau$ for (a) $\alpha=0.627$ and (b) $\alpha=0.25$ for the case of an asymmetric parabolic potential in Fig. 6, where the potential is set to zero at $t=15000 \tau$. The ion cluster remains tightly packed for $\alpha=0.627$, while the ion cloud expands for $\alpha=0.25$.

$t=20000 \tau$. Hence, the short-range potential around ions for $\alpha=0.627$ (cf. Fig. 2) lead to the solidification of ions, which is not the case for the the short-range repulsive potential for $\alpha=0.25$.

\section{Summary and conclusions}

In this paper, we have carried out particle simulations to demonstrate clustering of ions due to the newly found SEAF arising from collective interactions between an ensemble of degenerate electrons that shield ions in dense quantum plasmas. Specifically, the SEAF leads to clustering/condensation or coagulation of ions in the absence of an external confining potential for charged particles. However, ion clustering can be put on the firm footing by calculating the dynamical ion structure factor (DISF) based on the fluctuation-dissipation theorem and the dielectric constant of degenerate electrons and strongly correlated ions in a viscous quantum plasma. It may well turn out that the DISF will reveal longrange correlations between ions. It is our belief that the formation of ion nano-clusters is going to play a 
valuable role in the area of high-density compressed plasmas with degenerate electrons (Son and Fisch 2004, 2005, 2006; Malkin et al. 2007; Glenzer and Redmer 2009) for ICF to succeed, and also in the emerging field of nano-material sciences (e.g. nanodiodes, metallic nanostructures for thin films (Crouseilles et al. 2008), and nanowires), where closely packed ions will lend support to enhanced fusion probabilities (with anomalous fusion cross sections) for controlled thermonuclear ICF, and may also influence the electric properties (e.g. resistivity) of new high-density plasma materials at relatively high temperatures. Finally, we stress that the Cooper pairing of ions at atomic dimensions shall provide possibility of novel superconducting plasma-based nanotechnology, since the electron transport in nanostructures would be rapid due to shortened distances between ions in the presence of the novel SEAF.

\section{Acknowledgements}

This work was supported by the Deutsche Forschungsgemeinschaft through the project SH21/3-2 of the Research Unit 1048.

\section{References}

Akbari-Moghanjoughi, M. 2012 Shukla-Eliasson attractive force: revisited. J. Plasma Phys. pp. 1-8. doi:10.1017/ S0022377812000839, Published online: 30 October 2012.

Avinash, K. 2007 Mean-field theory of critical phenomenon for mutually repelling particles in complex plasmas. Phys. Rev. Lett. 98, 095003.

Barkan, A. and Merlino, R. L. 1995 Confinement of dust particles in a double layer. Phys. Plasmas 2, 3261-3265.

Berg, T. G. O. and Gaukler, T. A. 1969 Apparatus for the study of charged particles and droplets. Am. J. Phys. 37, 1013-1018.

Bohm, D. 1952 A suggested interpretation of the quantum theory in terms of "hidden" variables. I. Phys. Rev. 85 , 166-179.

Bohm, D. and Pines, D. 1953 A collective description of electron interactions: III. Coulomb interactions in a degenerate electron Gas. Phys. Rev. 92, 609-625.

Brey, L., Dempsey, J., Johnson, N. F. and Halperin, B. I. 1990 Infrared optical absorption in imperfect parabolic quantum wells. Phys. Rev. B 42, 1240-1247.

Brodin, G., Marklund, M. and Manfredi, G. 2008 Quantum plasma effects in the classical regime. Phys. Rev. Lett. 100, 175001.

Chandrasekhar, S. 1931 The maximum mass of ideal white dwarfs. Astrophys. J. 74, 81-82.

Chandrasekhar, S. 1939 An Introduction to the Study of Stellar Structure. Chicago, IL: The University of Chicago Press.

Chu, J. H. and I, L. 1994 Direct observation of Coulomb crystals and liquids in strongly coupled rf dusty plasmas. Phys. Rev. Lett. 72, 4009-4012.

Crandall, R. S. and Williams, R. 1971 Crystallization of electrons on the surface of liquid helium. Phys. Lett. A 34, 404-405.
Crouseilles, N., Hervieux, P. A. and Manfredi, G. 2008 Quantum hydrodynamic model for the nonlinear electron dynamics in thin metal films. Phys. Rev. B 78, 155412.

Debye, P. and Hückel, E. 1923 Zur Theorie der Electrolyte I: Gefrierpunktserniedrigung und verwandte Erscheinungen. Phys. Z. 24, 185-206.

Deshpande, V. V. and Bockrath, M. 2008 The one-dimensional Wigner crystal in carbon nanotubes. Nature Phys. 4, 314318.

Drewsen, M., Brodersen, C., Hornekær, L., Hangst, J. S. and Schiffer, J. P. 1998 Large ion crystals in a linear Paul trap. Phys. Rev. Lett. 81, 2878-2881.

Fermi, E. 1927 Un metodo statistico per la determinazione di alcune proprietà dell'atomo. Rend. Acad. Na. Lincei 6 , 602-607.

Fortov, V. E. 2009 Extreme state of matter on earth and in space. Phys. Usp. 52, 615-647.

Fortov V. E., Nefedov, A. P., Torchinsky, V. M., Molotkov, V. I., Petrov, O. F., Samarian, A. A., Lipaev, A. A. and Khrapak, A. G. 1997 Crystalline structure of strongly coupled dusty plasmas in dc glow discharge strata. Phys. Lett. A 229 , 317-322.

Gardner, C. L. and Ringhofer, C. 1996 Smooth quantum potential for the hydrodynamic model. Phys. Rev. E 53, 157-167.

Glenzer, S. H., Landen, O. L., Neumayer, P., Lee, R. W., Widmann, K., Pollaine, S. W., Wallace, R. J., Gregori, G., Holl, A., Bornath, T., et al. 2007 Observations of plasmons in warm dense matter. Phys. Rev. Lett. 98, 065002.

Glenzer S. H. and Redmer, R. 2009 X-ray Thomson scattering in high energy density plasmas. Rev. Mod. Phys. 81, 16251663.

Grimes, C. C. and Adams, G. 1979 Evidence for a liquid-tocrystal phase transition in a classical, two-dimensional sheet of electrons. Phys. Rev. Lett. 42, 795-798.

Haas, F. 2011 Quantum Plasmas: An Hydrodynamical Approach. New York: Springer.

Hayashi, Y. and Tachibana, K. 1994 Observation of Coulombcrystal formation from carbon particles grown in a methane plasma. Jpn. J. Appl. Phys. 33, L804-L806.

Hedin, L. and Lundqvist, B. I. 1971 Explicit local exchangecorrelation potentials. J. Phys. C: Solid State Phys. 4, 2064 2083.

Klimontovich, Yu. L. and Silin, V. P. 1952a Dokl. Akad. Nauk SSSR, 82, 361.

Klimontovich, Yu. L. and Silin, V. P. 1952b Concerning the spectra of systems of interacting particles. Zh. Eksp. Teor. Fiz. 23, 151-160.

Klumov, B. 2010 On melting criteria for complex plasma. Phys. Usp. 53, 1053-1065.

Kremer, K., Robbins, M. O. and Grest, G. S. 1986 Phase diagram of Yukawa systems: model for charge-stabilized colloids. Phys. Rev. Lett. 57, 2694-2697.

Landau, L. D. and Lifshitz, E. M. 1980 Statistical Physics. Oxford, UK: Butterworth-Heinemann.

Langmuir, I. 1929 The interaction of electron and positive ion space charges in cathode sheaths. Phys. Rev. 33, 954-989.

Malkin, V. M., Fisch, N. J. and Wurtele, J. S. 2007 Compression of powerful x-ray pulses to attosecond durations by stimulated Raman backscattering in plasmas. Phys. Rev. E 75, 026404.

Manfredi, G. 2005 How to model quantum plasmas. Fields Inst. Commun. 46, 263-287. 
Manfredi, G. and Haas, F. 2001 Self-consistent fluid model for a quantum electron gas. Phys. Rev. B 64, 075316.

Melrose, D. B. 2008 Quantum Plasmadynamics: Unmagnetized Plasmas. Berlin, Germany: Springer.

Mendonça, J. T. 2011 Wave kinetics of relativistic quantum plasmas. Phys. Plasmas 18, 062101.

Mohideen, U., Rahman, H. U., Smith, M. A., Rosenberg, M. and Mendis, D. A. 1998 Intergrain coupling in dusty-plasma Coulomb crystals. Phys. Rev. Lett. 81, 349-352.

Mølhave, K. and Drewsen, M. 2000 Formation of translationally cold $\mathrm{MgH}^{+}$and $\mathrm{MgD}^{+}$molecules in an ion trap. Phys. Rev. A 62, 011401.

Nambu, M., Vladimirov, S. V. and Shukla, P. K. 1995 Attractive forces between charged particulates in plasmas. Phys. Lett. A 203, 40-42.

Resendes, D. F., Mendonça, J. T. and Shukla, P. K. 1998 Formation of dusty plasma molecules. Phys. Lett. A 239, 181-186.

Robertson, S. and Younger, R. 1999 Coulomb crystals of oil droplets. Am. J. Phys. 67, 310-315.

Salpeter, E. E. 1961 Energy and pressure of a zero-temperature plasma. Astrophys. J. 134, 669-682.

Shaikh D. and Shukla, P. K. 2007 Fluid turbulence in quantum plasmas. Phys. Rev. Lett. 99, 125002.

Shukla, P. K., Akbari-Moghanjoughi, M. and Eliasson B. 2012 Comment on "On 'Novel attractive forces' between ions in quantum plasmas - failure of linearized quantum hydrodynamics.” arXiv:1206.3456 [physics.plasm-ph].

Shukla, P. K. and Eliasson, B. 2006 Formation and dynamics of dark solitons and vortices in quantum electron plasmas. Phys. Rev. Lett. 96, 245001.

Shukla, P. K. and Eliasson, B. 2007 Nonlinear interactions between electromagnetic waves and electron plasma oscillations in quantum plasmas Phys. Rev. Lett. 99, 096401.

Shukla, P. K. and Eliasson, B. 2009 Fundamentals of dust plasma interactions. Rev. Mod. Phys. 81, 25-50.

Shukla, P. K. and Eliasson, B. 2010 Nonlinear aspects of quantum plasma physics. Phys. Usp. 53, 51-76.

Shukla, P. K. and Eliasson, B. 2011 Nonlinear collective interactions in quantum plasmas with degenerate electron fluids. Rev. Mod. Phys. 83, 885-906.

Shukla, P. K. and Eliasson B. 2012a Novel attractive force between ions in quantum Plasmas. Phys. Rev. Lett. 108, 165007.

Shukla, P. K. and Eliasson B. 2012b Erratum: novel attractive force between ions in quantum plasmas. Phys. Rev. Lett. 108, 165007 (2012)]; Phys. Rev. Lett. 108, 219902(E).

Shukla, P. K. and Eliasson B. 2012c Erratum: novel attractive force between ions in quantum plasmas. Phys. Rev. Lett. 108, 165007; Phys. Rev. Lett. 109, 019901(E).
Shukla, P. K. and Mamun, A. A. 2002 Introduction to Dusty Plasma Physics. Bristol, UK: Institute of Physics.

Shukla, P. K. and Rao, N. N. 1996 Coulomb crystallization in colloidal plasmas with streaming ions and dust grains. Phys. Plasmas 3, 1770-1772.

Son S. and Fisch, N. J. 2004 Aneutronic fusion in a degenerate plasma. Phys. Lett. A 329, 76-82.

Son S. and Fisch, N. J. 2005 Current-drive efficiency in a degenerate plasma. Phys. Rev. Lett. 95, 225002.

Son S. and Fisch, N. J. 2006 Controlled fusion with hotion mode in a degenerate plasma. Phys. Lett. A 356, 6571.

Staanum, P. F., Højbjerre, K., Skyt, P. S., Hansen A. K. and Drewsen, M. 2010 Rotational laser cooling of vibrationally and translationally cold molecular ions. Nature Phys. 6, 271-274.

Tan, J. N., Bollinger, J. J., Jelenkovic, B. and Wineland, D. J. 1995 Long-range order in laser-cooled, atomic-ion Wigner crystals observed by Bragg scattering. Phys. Rev. Lett. 75, 4198-4201.

Thomas, L. H. 1927 The calculation of atomic fields. Math. Proc. Cambridge Phil. Soc. 23, 542-548.

Thomas, H., Morfill, G. E., Demmel, V., Goree, J., Feuerbacher, B. and Möhlmann, D. 1994 Plasma crystal: Coulomb crystallization in a dusty plasma. Phys. Rev. Lett. 73, 652655 .

Tsintsadze, N. L. and Tsintsadze, L. N. 2009 Novel quantum kinetic equations of the Fermi particles. Europhys. Lett. 88, 35001.

Vladimirov, S. V. and Nambu, M. 1995 Attraction of charged particulates in plasmas with finite flows. Phys. Rev. E 52, R2172-R2174.

Vladimirov, S. V. and Tyshetskiy, Yu. O. 2011 On description of a collisionless quantum plasma. Phys. Usp. 54, 1243-1256.

Watanabe, H. 1956 Experimental evidence for the collective nature of the characteristic energy loss of electrons in solidsStudies on the dispersion relation of plasma frequency. J. Phys. Soc. Jpn. 11, 112-119.

Wigner, E. 1934 On the interaction of electrons in metals. Phys. Rev. 46, 1002-1011.

Wilhelm, H. E. 1971 Wave-mechanical formulation of plasma dynamics in longitudinal electric fields. Z. Phys. 241, 1-8.

Winter H. and Ortjohann, H. W. 1991 Simple demonstration of storing macroscopic particles in a "Paul trap." Am. J. Phys. 59, 807-813.

Wuerker, R. F., Shelton, H. and Langmuir, R. V. 1959 Electrodynamic containment of charged particles. J. Appl. Phys. 30, 342-349.

Yukawa H. 1935 On the interaction of elementary particles I. Proc. Phys. Math. Jpn. 17, 48-57. 